\title{
Allocation and Sizing of the Distributed Generation for Power System Instability Prevention
}

\author{
Dhafer Mayoof Dahis \\ \{ Eng.dafir@stu.edu.iq ${ }^{1}$ \} \\ Southern Technical University Basrah ,Iraq ${ }^{1}$
}

\begin{abstract}
Supplying customers regularly and network stability are some of the most essential tasks for power suppliers companies. In this paper, distributed generation resources (DGs) are used to increase the stability of the transmission network when the most critical line is eliminated. One of the major works in this paper is calculating the revenue of the network operators due to a reduction in purchasing real power from generation companies since using distributed generation resources. Moreover, the cost of these resources and the cost of environmental pollution, are all considered in this study. Categorizing the load into the three-time span, light, medium, and peak load, with variation in consumption, along with considering variation in purchasing power tariffs in the mentioned category, inflation rates, and annual interest rates, and the proper lifetime expectancy of distributed generation resources are other significant aspects of this paper. Mat-Power, in MATLAB, is used for load power flow equations and other network computations to find the optimum location and capacity of distributed resources. A 14 IEEE bus Reliability Test System is simulated to indicate the network stability of the power system in the presence of distributed generation sources.
\end{abstract}

Keywords: Priority of transmission lines; Distributed generation resources; Optimum allocation and sizing, Timespan, Interest rates, and inflation.

\section{Introduction}

Power system operation is done in the safest mode so that the elimination of each power line cannot create any obstacle in a normal operating state of the power system. To assess the safety of the power system, it is necessary to simulate the elimination of each power line to study power system performance in terms of voltage deviation for each load point and line overload [1]. Therefore, identifying the most critical power line, the line that its elimination either deliberately or unknowingly takes the power system more quickly to instability, over other lines is significantly essential. On the other hand, due to the competitive market among electricity industries, high and unprofitable investments are eradicated, and consequently, keeping the power system stable and secure is accompanied by a lot of stress $[2,3]$.

Furthermore, the restructuring of the electricity industries changed the rules and economic criteria in the power system [4]. Applying distribution Generations (DGs) in power systems is one of the prominent phenomena that play an essential role in power system restructuring [5]. In this paper, the DG is meant to be a diesel generator unit with a quick launch in the 
transmission network. The rapid loading rate of these resources is considered to be their significant benefit.

In most researches on the location and optimal sizing of distributed generations, these resources are addressed in Low and Medium-voltage (LV and MV), and the placement of DGs in the transmission network is rarely taken into consideration. In [6], the priority of the lines is based on the bus voltage only; due to the elimination of each of the lines various constraints of the power system are at risk and the prioritization of the lines cannot be considered from only one perspective. In [7], optimal allocation and sizing of distributed generation resources are aimed at reducing losses. In [8], placing DGs in the transmission and distribution system for proper scheduling the interruption of the loads, while interrupting the load should be the last way to prevent instability in the power system. In [9], the installation of distributed generation resources in the distribution system is done by considering the increase in voltage of the standard coupling points and the thermal constraints of the power lines .

In this paper, distributed generation resources are implemented to increase power system stability. Given the economic aspects of installing these resources, the optimal DGs allocation and sizing determination is significantly essential. This article generally has two stages:

- First step: power lines prioritization.

- The second step: optimal allocation and sizing determination of distributed generation sources to prevent the power system from instability after the most critical line identification and then elimination.

Significant features of this article include:

- Considering the costs of distributed generation resources along with the cost of environmental pollution.

- Considering the gained profit for the operators due to injecting real power via DGs and consequently reduction in purchasing power.

- Considering three-time span, light, medium, and peak load, with variation in consumption levels and variable purchasing power tariffs in specified periods.

- Consider the life expectancy for distributed generation resources along with inflation rate and annual interest rate.

- Simultaneous optimal allocation and sizing determination of DGs to make the simulation more realistic.

\section{Prioritization of lines in the transmission network}

In prioritizing the power line using specific performance indicators the effect of eliminating each power line on the entire system is described. An overview of the impact of cutting each of the power lines on power system operators is provided. Prioritization took place while indicators are arranged in numerical order and events that have a more extensive index creates more problems for the power system. The following two hands are presented in the following.

\subsection{Real Power Flow Index}

Consider a power system that works in a stable condition. If one or more transmission lines are eliminated other power lines will face overloading and these phenomena put the power system 
security and stability in danger. They can lead the power system into unstable conditions. Real power flow index as presented in (1) [10].

$$
P I w_{i}=\sum_{j=\text { all other branches }}\left(\frac{P_{\text {flowj }}}{P_{j}^{\max }}\right)^{2 n}
$$

Where:

$P I w_{i}$ is efficiency related to the real power flow index when the transmission line is eliminated. $P_{\text {flow } j}$ is power flow in the transmission line $\mathrm{j}^{\text {th }}$, after the $\mathrm{i}^{\text {th }}$ transmission line is eliminated.

$P_{j}^{\max }$ is Maximum transmittable power in $\mathrm{j}^{\text {th }}$ power line

$\mathrm{n}$ is an arbitrary natural number

\section{Voltage Performance Index}

After each line is eliminated, the voltage of every point of the power system faces variation, and consequently, there is a risk of a voltage security violation. Voltage deviation in the acceptable range is expressed by the voltage performance index in (2). This indicator represents the difference between the bus voltages before and after the power lines elimination relevant to the maximum allowable voltage deviation [10].

$$
P I v_{i}=\sum_{k \in a}\left(\frac{\left(V_{k}^{\text {pre }}-V_{k}^{\text {past }}\right)}{\max \text { vol drop }}\right)^{2 n}
$$

In the above equation:

$P I v_{i}$ is power system voltage performance index when $\mathrm{i}^{\text {th }}$ power line is eliminated.

$a$ is set buses whose voltage is beyond the limitation.

$V_{k}^{\text {pre }}$ is $\mathrm{k}^{\text {th }}$ bus voltage before $\mathrm{i}^{\text {th }}$ transmission line is eliminated.

$V_{k}^{\text {past }}$ is $\mathrm{k}^{\text {th }}$ bus voltage after $\mathrm{i}^{\text {th }}$ transmission line is eliminated.

\section{Allocation and sizing of the distributed generation resources}

Allocation and inappropriate sizing determination for distributed generation resources not only create more cost for the power system but also lead to undesirable effects on system performance [11]. Determining the allocation and optimal sizing of distributed generation in the transmission network can be presented as an optimization problem.

\subsection{The objective function}

Equation (3) express the objective function of the problem while its details are presented in equations (4) to (15). 


\section{$\operatorname{Max}$ Benefit-Cost ${ }_{\text {total }}$}

Where:

Benefit represents the benefit of the system operators by reducing the consumed power from power plants.

Cost $_{\text {total }}$ Indicates the total charges imposed on the power system operators.

The Benefit function is computed from (4).

$$
\text { Benefit }=B P V\left(B_{1}\right)
$$

In the above equation:

BPV (B1) expresses the gained revenue from the reduction of the purchasing power from power plants over the proper lifetime of DG and is calculated from equation (5) [12].

$\operatorname{BPV}\left(B_{1}\right)=B_{1} \times \sum_{t=1}^{T_{\text {total }}}\left(\frac{1+\operatorname{InfR}}{1+\operatorname{Int} R}\right)^{t}$

In the above equation:

$T_{\text {total }}$ is a proper lifetime of DG

$\operatorname{InfR}$ is the annual inflation rate.

IntR is the annual interest rate.

B1 is computed annual profit due to the reduction in the purchasing power from power plants, which is calculated from equation (6).

$B_{1}=\sum_{j=1}^{3} C_{j}^{P} \times \Delta$ Ploss $_{j} \times T_{j}$

In the above equation:

$j=1,2,3$ expresses three light, medium, and peak load span times.

$C_{j}^{P}$ is the cost of purchased active power from power plants.

$T_{j}$ is duration related to the $\mathrm{jth}$ period.

$\Delta$ Ploss $_{j}$ are losses of active power variation due to the absence and presence of DG in the $\mathrm{j}^{\text {th }}$ time which can be calculated from equation (7).

$$
\Delta \operatorname{Ploss}_{j}=\operatorname{Ploss}_{j}^{\text {NoDG }}-\text { Ploss }_{j}^{\text {With DG }}
$$

In the above equation:

Ploss $_{j}^{\text {No DG }}$ is active power losses without the presence of the DG at the jth time.

Ploss ${ }_{j}^{\text {With DG }}$ is active power losses insight of the DG at the $\mathrm{j}^{\text {th }}$ time. Function related to 
Cost $_{\text {total }}$ can be calculated from (8).

$\mathrm{Cost}_{\text {total }}=\left(\begin{array}{l}\operatorname{Cost}_{D G-\text { Investment }}+C P V\left(C_{1}\right) \\ +C P V\left(C_{2}\right)+C P V\left(C_{3}\right)\end{array}\right)$

$\operatorname{Cos} t_{D G-\text { Investment }}$ is DG investment cost which can be computed from (9).

$\operatorname{Cos} t_{D G-\text { Investment }}=k_{D G-I n v} \times \sum_{i=1}^{N_{B U S}}\left[P_{D G_{i}}\right]$

Where:

$k_{D G-I n v}$ is investment cost for DG for generating $1 \mathrm{MW}$ of power per 1 Hour. $P_{D G_{i}}$ is the generated power from DG which is connected to the $\mathrm{i}^{\mathrm{th}}$ bus.

$N_{B U S}$ is a number of buses in a simulated power system $C P V\left(C_{1}\right)$ represents the current value of the operation cost for DG, which is obtained from equation (10) [10].

$C P V\left(C_{1}\right)=\operatorname{Cost}_{\text {DG-Maintenance }} \times \sum_{t=1}^{T_{\text {total }}}\left(\frac{1+\operatorname{InfR}}{1+\operatorname{IntR}}\right)^{t}$

Where:

Cost $_{\text {DG-Maintenance }}$ represents annual cost maintenance for DG from eq. (11)

$\operatorname{Cost}_{\text {DG-Maintenance }}=k_{\text {DG-Maintenance }} \times T \times \sum_{i=1}^{N_{B U S}} P_{D G_{i}}$

$\mathrm{k}_{\text {DG-Maintenance }}$ is the cost of maintenance for DG for generating 1MW per hour

$C P V\left(C_{2}\right)_{\text {is }}$ present value for DG operational cost and comes from eq. (12)

$C P V\left(C_{2}\right)=$ Cost $_{\text {DG-Operation }} \times \sum_{t=1}^{T_{\text {total }}}\left(\frac{1+\operatorname{InfR} R}{1+\operatorname{IntR}}\right)^{t}$

Where:

Cost $_{\text {DG-Operation }}$ is the DG annual operation cost computable using (13) [12].

$\operatorname{Cost}_{\text {DG-Operation }}=k_{\text {DG-Operation }} \times T \times \sum_{i=1}^{N_{B U S}} P_{D G_{i}}$

In the above equation:

$k_{\text {DG-Operation }}$ is DG cost of operating per megawatt-hour

$C P V\left(C_{3}\right)$ is the current value of environmental pollution caused by DGs, which is obtained from equation (14). 


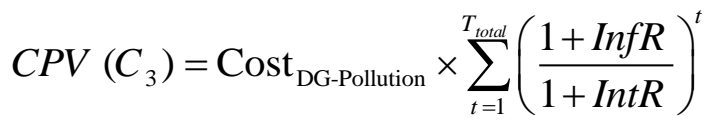

In the above equation:

Cost $_{\text {DG-Pollution }}$ shows the annual cost of environmental pollution caused by DG and is obtained from equation (15) [12].

$\operatorname{Cost}_{\text {DG-Pollution }}=T \times \sum_{i=1}^{N_{B U S}}\left[\left(\begin{array}{l}\rho_{\mathrm{NO}_{x}} \times E F_{\mathrm{NO}_{x}}+ \\ \rho_{\mathrm{SO}_{2}} \times E F_{\mathrm{SO}_{2}}+ \\ \rho_{\mathrm{CO}_{2}} \times E F_{\mathrm{CO}_{2}}\end{array}\right) \times P_{D G_{i}}\right]$

Where:

$\rho_{x}$ is penalty price for producing x-type of pollutants.

\subsection{Constraints on the issue}

The voltage security limitation is expressed by (16).

$\left|V_{\text {min }}\right| \leq\left|V_{i}\right| \leq\left|V_{\max }\right| \quad i=1,2, \ldots, \mathrm{N}_{\text {BUS }}$

The thermal constraint of the feeders is expressed using equation (17).

$\left|S_{i}\right| \leq \alpha\left|S_{i}^{\max }\right| \quad i=1,2, \ldots, \mathrm{N}_{\text {Branch }}$

Where:

$\mathrm{N}_{\text {Branch }}$ Number of system branches

$\alpha$ reliability index for power line congestion.

Generation capacity limitation for each DG is expressed in (18).

$P_{D G_{i}} \leq P_{D G}^{\max } \quad i=1,2, \ldots, \mathrm{N}_{\mathrm{BUS}}$

The limitation of reactive power generation using DG is given by (19)

$Q_{D G_{i}}^{\min } \leq Q_{D G_{i}} \leq Q_{D G_{i}}^{\max } \quad i=1,2, \ldots, \mathrm{N}_{\text {BUS }}$

Limitation related to a maximum total capacity of DGs is expressed in (20).

$\sum_{i=1}^{N_{B U S}} P_{D G_{i}} \leq P_{D G}^{\text {Max -Total }}$ 


\section{Simulation results}

\subsection{Power lines prioritization}

Using the mentioned indices expressed in Section 2, are a deterministic factor for power lines prioritization. In this study, the vulnerability of transmission lines is considered, in fact, by applying the performance indices, the effect of each power line's elimination on other lines in the transmission system is examined. The modified load in light, medium, and peak loading span time are shown in Table 1.

Table 1. Modified load for an IEEE 14 bus RTS.

\begin{tabular}{cllllll}
\hline & \multicolumn{2}{l}{ Peak load } & \multicolumn{2}{l}{ Medium load } & \multicolumn{2}{l}{ Light load } \\
Bus & P & Q & \multicolumn{1}{l}{ P } & Q & \multicolumn{1}{l}{ Q } & \multicolumn{2}{l}{ Q } \\
& (MW) & (MVAr) & \multicolumn{1}{l}{ (MW) } & (MVAr) & (MW) & (MVAr) \\
\hline 1 & 0 & 0 & 0 & 0 & 0 & 0 \\
2 & 27.5 & 16.5 & 26.25 & $15 / 75$ & 25 & 15 \\
3 & 104.5 & 22 & 99.75 & 21 & 95 & 20 \\
4 & 55 & -4.4 & 52.5 & -4.2 & 50 & -4 \\
5 & 11 & 5.5 & 10.5 & 5.25 & 10 & 5 \\
6 & 16.5 & 11 & 15.75 & 10.5 & 15 & 10 \\
7 & 0 & 0 & 0 & 0 & 0 & 0 \\
8 & 0 & 0 & 0 & 0 & 0 & 0 \\
9 & 33 & 16.5 & 31.5 & 15.75 & 30 & 15 \\
10 & 11 & 16.5 & 10.5 & 15.75 & 10 & 15 \\
11 & 33 & 5.5 & 31.5 & 5.25 & 30 & 5 \\
12 & 44 & 16.5 & 42 & 15.75 & 40 & 15 \\
13 & 60.5 & 33 & 57.75 & 31.5 & 55 & 30 \\
14 & 33 & 16.5 & 31.5 & 15.75 & 30 & 15 \\
\hline
\end{tabular}

To determine the efficiency index in equation (1), $\mathrm{n}=2$ is considered, and the maximum transmitted power of each line is assumed to be $100 \mathrm{MW}$. In equation (2), $n=1$ is also considered. In Table 2, ten lines are identified which their elimination leads to the highest risk for the power system. Elimination of the $10^{\text {th }}$ and $13^{\text {th }}$ power lines leads to divergence in power load flow. According to the objectives of this paper elimination of the power lines that create a divergence in power load flow equations is excluded. Therefore Line 2 is the most critical in this power system. 
Table 2. Prioritization of transmission lines based on actual power flow and voltage performance induces

\begin{tabular}{llllll}
\hline Rank & Line & $\begin{array}{l}\text { From } \\
\text { bus }\end{array}$ & $\begin{array}{l}\text { To } \\
\text { bus }\end{array}$ & PIv & PIw \\
\hline 1 & 2 & 1 & 5 & 10.2 & 118.16 \\
2 & 3 & 2 & 3 & 7.9 & 105.19 \\
3 & 17 & 9 & 14 & 6.6 & 104.04 \\
4 & 12 & 6 & 12 & 6.3 & 104.04 \\
5 & 16 & 9 & 10 & 5.8 & 96.04 \\
6 & 11 & 6 & 11 & 5.1 & 92.16 \\
7 & 14 & 7 & 8 & 3.9 & 88.36 \\
8 & 9 & 4 & 9 & 3.5 & 88.36 \\
9 & 1 & 1 & 2 & 2.4 & 86.49 \\
10 & 5 & 2 & 5 & 1.2 & 82.81 \\
\hline
\end{tabular}

\section{6 location and optimal sizing for distributed generation resources}

According to the calculations carried out in the first stage, the calculations in this section are carried out without the presence of line number 2. The distributed generation resources are considered as negatively load with the ability to generate reactive and reactive power, and the maximum capacity for each DG is 80 megawatts. The parameters considered for the optimization problem are presented in Table (3).

Table 3 Input Parameters.

\begin{tabular}{ccc}
\hline Parameters & Unit & Value \\
\hline$k_{D G-I n v}$ & $\$ / M W$ & 3800 \\
$k_{\text {DG-Maintenance }}$ & $\$ / M W h$ & 0.0075 \\
$k_{\text {DG-Operation }}$ & $\$ / M W h$ & 0.08 \\
$T$ & hour & 8760 \\
$S_{i}^{\max }$ & $M V A$ & 100 \\
$\left|V_{\min }\right|$ & $P . U$. & 0.95 \\
$\left|V_{\max }\right|$ & $P . U$. & 1.05 \\
$P_{D G}^{M a x-T o t a l}$ & $M W$ & 2500 \\
$Q_{D G_{i}}^{\min }$ & $M V A r$ & -26.2947 \\
$Q_{D G_{i}}^{\max }$ & $M V A r$ & +26.2947 \\
$T_{\text {total }}$ & year & 20 \\
\hline
\end{tabular}




\begin{tabular}{ccc}
\hline InfR & $\%$ & 8.5 \\
IntR & $\%$ & 13 \\
$\cos \varphi_{D G}$ & - & 0.95 \\
$\alpha$ & $\%$ & 95 \\
\hline
\end{tabular}

The different periods and tariffs for purchasing active power from power plants in a light, medium, and peak loading are given in Table (4). The values for environmental pollution are described in Table 5. Table (6) presents the results related to optimum allocation and sizing of the DGs.

Table 4. Data for a three-time span.

\begin{tabular}{|c|c|c|}
\hline Time span & $\begin{array}{l}\text { Period } / \text { year) } \\
\text { (hour }\end{array}$ & Price $(\$ / M W h)$ \\
\hline Light load & 2500 & 0.54 \\
\hline Medium load & 5010 & 0.85 \\
\hline Peak load & 1250 & 1.02 \\
\hline
\end{tabular}

Table 5. Environmental Pollution Data [13].

\begin{tabular}{ccc}
\hline $\begin{array}{c}\text { Type of } \\
\text { pollutant }\end{array}$ & $\begin{array}{c}\text { Pollution coefficient } \\
(\mathrm{lb} / \mathrm{MWh})\end{array}$ & $\begin{array}{c}\text { Cost of pollution } \\
(\$ / \mathrm{lb})\end{array}$ \\
\hline $\mathrm{NO}_{x}$ & 0.00044 & 4.2 \\
$\mathrm{SO}_{2}$ & 0.000008 & 0.99 \\
$\mathrm{CO}_{2}$ & 0.001596 & 0.014 \\
\hline
\end{tabular}

The voltage profile in light, medium, and peak loading periods is illustrated in Fig. 1. Moreover, active transmitted power in lines by considering different time spans in either presence or absence of the DGs and active and reactive losses are given in Table 7 and Table 8, respectively. Table (9) illustrates the profits and costs from DGs installation. 


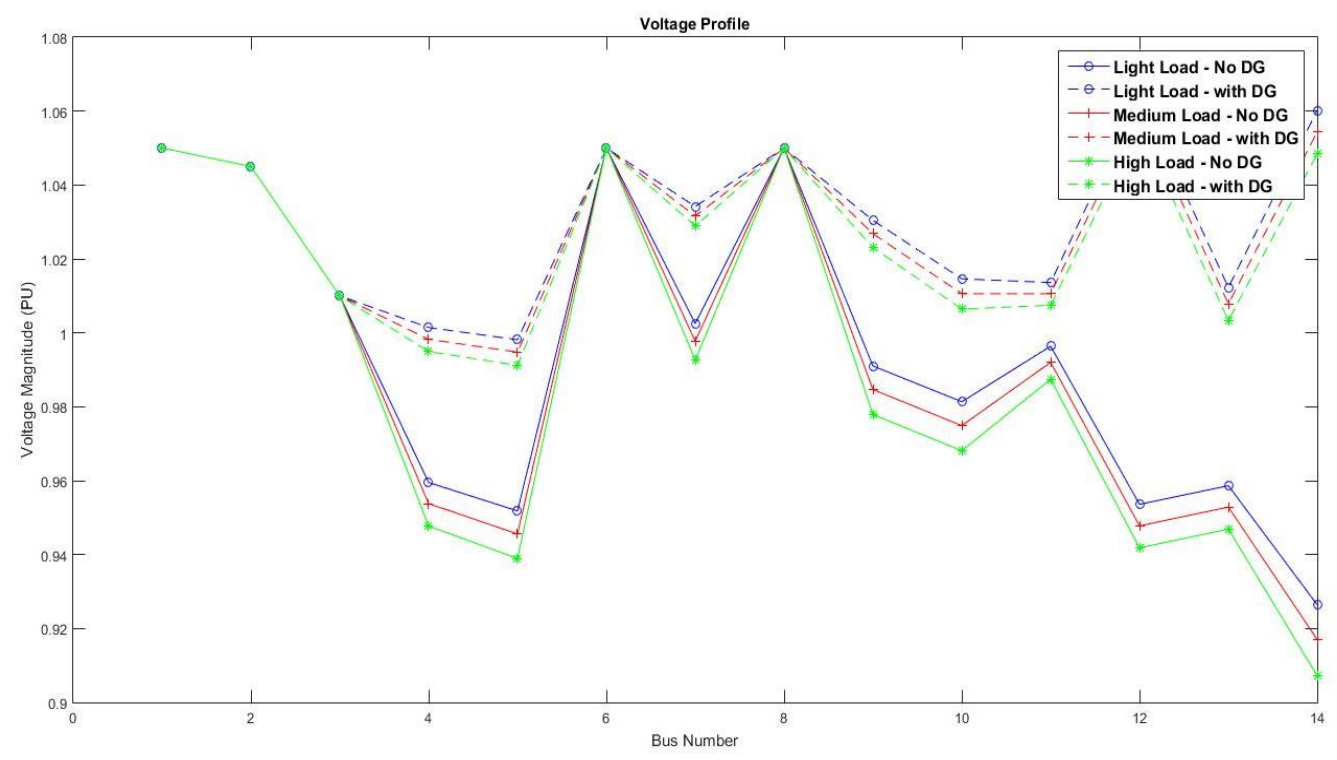

Fig 1. Voltage profile absence and presence of the DGs.

Table 6. Optimal Allocation and sizing of the DGs.

\begin{tabular}{ccc}
\hline Bus & $\mathrm{P}(\mathrm{MW})$ & $\mathrm{Q}(\mathrm{MVAr})$ \\
\hline 3 & 11.856 & 3.896 \\
12 & 69.149 & 22.728 \\
14 & 69.935 & 22.986 \\
\hline
\end{tabular}

Table 7. Actual transmitted power in lines.

\begin{tabular}{|c|c|c|c|c|c|c|c|c|}
\hline \multirow{2}{*}{ Line } & \multirow{2}{*}{$\begin{array}{l}\text { Fro } \\
\mathrm{m} \\
\text { bus }\end{array}$} & \multirow{2}{*}{$\begin{array}{l}\text { To } \\
\text { bu } \\
\text { s }\end{array}$} & \multicolumn{2}{|c|}{$\begin{array}{l}\text { Peak Load } \\
\text { (MW) }\end{array}$} & \multicolumn{2}{|c|}{$\begin{array}{l}\text { Medium load } \\
(\mathrm{MW})\end{array}$} & \multicolumn{2}{|c|}{$\begin{array}{l}\text { Light load } \\
\text { (MW) }\end{array}$} \\
\hline & & & $*$ & $* *$ & $*$ & $* *$ & $*$ & $* *$ \\
\hline 1 & 1 & 2 & 182.1 & 6.4 & 156 & 27.7 & 130 & 48.5 \\
\hline 2 & 2 & 3 & 0 & 0 & 0 & 0 & 0 & 0 \\
\hline 3 & 2 & 4 & 157.1 & 90 & 149 & 83 & 141 & 76 \\
\hline 4 & 2 & 5 & 158.3 & 86 & 150 & 78.9 & 142 & 71.9 \\
\hline 5 & 3 & 4 & 20.8 & 6.1 & 19 & 7.07 & 17.8 & 7.86 \\
\hline 6 & 4 & 5 & 1.33 & 19 & 0.64 & 19.1 & 0.03 & 19 \\
\hline 7 & 4 & 7 & 68.56 & 27 & 65.4 & 24.6 & 62.2 & 21.6 \\
\hline 8 & 4 & 9 & 38.76 & 16 & 37.1 & 14.1 & 35.3 & 12.4 \\
\hline 9 & 5 & 6 & 135 & 52 & 128 & 46 & 121 & 40.1 \\
\hline
\end{tabular}




\begin{tabular}{lllllllll}
10 & 6 & 11 & 11.26 & 19 & 10.6 & 19.1 & 9.97 & 18.5 \\
11 & 6 & 12 & 41.15 & 5.6 & 39.1 & 7.42 & 37 & 9.15 \\
12 & 6 & 13 & 66.13 & 21 & 62.7 & 18.5 & 59.4 & 15.7 \\
13 & 7 & 8 & 67.07 & 22 & 58.5 & 33.4 & 80.9 & 60.8 \\
14 & 7 & 9 & 68.56 & 27 & 65.4 & 24.6 & 62.3 & 21.6 \\
15 & 9 & 10 & 34.68 & 25 & 33.1 & 23.6 & 31.5 & 22.1 \\
16 & 9 & 14 & 39.65 & 15 & 37.9 & 16.3 & 36.1 & 18.1 \\
17 & 10 & 11 & 23.28 & 14 & 22.2 & 12.9 & 21.2 & 11.9 \\
18 & 12 & 13 & 5.47 & 19 & 5.28 & 19.7 & 5.08 & 19.9 \\
19 & 13 & 14 & 4.10 & 21 & 4.11 & 20.9 & 4.10 & 20.7 \\
$*:$ Without DG & & & **: With DG & & & \\
\hline
\end{tabular}

Table 8. Active and reactive power losses in lines.

\begin{tabular}{|c|c|c|c|c|c|c|}
\hline \multirow[b]{2}{*}{ Losses } & \multicolumn{2}{|c|}{ Peak Load } & \multicolumn{2}{|c|}{ Medium Load } & \multicolumn{2}{|c|}{ Peak Load } \\
\hline & $\begin{array}{c}\text { With } \\
\text {-out } \\
\text { DG }\end{array}$ & $\begin{array}{l}\text { With } \\
\text { DG }\end{array}$ & $\begin{array}{c}\text { With } \\
\text {-out } \\
\text { DG }\end{array}$ & $\begin{array}{l}\text { With } \\
\text { DG }\end{array}$ & $\begin{array}{c}\text { With } \\
\text {-out } \\
\text { DG } \\
\end{array}$ & $\begin{array}{l}\text { With } \\
\text { DG }\end{array}$ \\
\hline $\begin{array}{l}P_{\text {Loss }} \\
\text { (MW) }\end{array}$ & 53 & 15.4 & 46.4 & 13.7 & 40.5 & 12.3 \\
\hline $\begin{array}{l}\text { QLoss } \\
\text { (MVAr) }\end{array}$ & 194 & 18.3 & 166.7 & 10.3 & $\begin{array}{c}141 . \\
4\end{array}$ & 3.8 \\
\hline
\end{tabular}

Table 9. Review of Economic Measures.

\begin{tabular}{ccc}
\hline \multicolumn{2}{c}{ Type } & Value (\$) \\
& Benefit & 3025900 \\
\hline \multirow{2}{*}{ Cost } & Investment & 573570 \\
& Maintenance & 133030 \\
& Operation & 1419000 \\
& Environmental & 33315 \\
& pollution & 2158915 \\
\hline
\end{tabular}

\section{Conclusion}

Simulation results indicate in presence of the distributed generation resources (DGs), system stability is achievable. According to Figure (1) and Table (7), optimum allocation and sizing of DGs cause voltage security limitation and power line congestion for different periods. Table (8) also shows a significant reduction in active and reactive power losses over other time spans in presence of the DGs. Furthermore, with the reduction of active and reactive power losses due to the presence of the DGs, system operators can gain a prominent revenue. As a result, installing these resources will be economically justifiable. 


\section{References}

[1] M. Amohadi and M. Fotuhi-Firuzabad, "Optimal Placement of Automatic Switching Equipment in Radial Distribution Networks Based on Protective Coordination", Journal of Electrical Engineering \& Technology, vol. 14, no. 3, pp. 1127-1137, 2019.

[2] Ravishankar, Vijayendra, Veeramanju, "Voltage and power losses control using distributed generation and computational intelligence", Tehnicki vjesnik - Technical Gazette, vol. 23, no. 4, 2016.

[3] P. Pavani and S. Singh, "Placement of DG for reliability improvement and loss minimization with reconfiguration of radial distribution systems", International Journal of Energy Sector Management, vol. 8, no. 3, pp. 312-329, 2014.

[4] L. Jian, Z. Qian, Z. Liangang and Y. Mengkai, "Distributed economic dispatch method for power system based on consensus", IET Renewable Power Generation, vol. 14, no. 9, pp. 1424-1432, 2020.

[5] Marko Vukobratovic, Predrag Maric, "Evaluation of Distributed Generation Impact on Reliability of a Distribution System using DIg SILENT Power Factory", Regular, vol. 9, no. 10, pp. 381-389, 2020.

[6] U. Sultana, A. Khairuddin, B. Sultana, N. Rasheed, S. Qazi and N. Malik, "Placement and sizing of multiple distributed generation and battery swapping stations using grasshopper optimizer algorithm", Energy, vol. 165, pp. 408-421, 2018.

[7] O. Khoubseresht and H. Shayanfar, "The role of demand response in optimal sizing and siting of distribution energy resources in distribution network with time-varying load: An analytical approach", Electric Power Systems Research, vol. 180, p. 106100, 2020.

[8] . Kaushik Das, Antonios Nitsas, Mufit Altin, Anca D Hansen, Poul E. Sorensen, "Improved Loadshedding Schems Considering Distributed Generation", IEEE Manchester, PowerTech, June 2017

[9] Desmond Okwabi Ampofo, Isaac Kofi Otchere, Emmanuel Asuming Frimpong, "An Investigative Study on Penetration Limits of Distributed Generation on Distributed Network", IEEE PES, PowerAfrica, pp. 573-576, June 2017

[10] Z. Ghofrani-Jahromi, M. Abedi, G. Gharehpetian and M. Masjed-Jamei, "contingency ranking using analytical hierarchry process for calculating bus weighting factors ", International Journal of Power and Energy Systems, vol. 30, no. 2, 2010

[11] Hijun Xing, Xin Sun, "Distributed Generation Locating and Sizing in Active Distribution Network Considering Network Reconfiguration”, IEEE Access, Vol 5, pp. 14768-14774, July 2017

[12] N. Khalesi, N. Rezaei, M.-R. Haghifam, "DG allocation with application of dynamic programming for loss reduction and reliability improvement," ELSEVIER, Vol.33, No.2, pp. 288-295, 2011

[13] G. Memarzadeh and F. Keynia, "A new index-based method for optimal DG placement in distribution networks", Engineering Reports, vol. 2, no. 10, 2020. 